\title{
A perturbation theory for large deviation functionals in fluctuating hydrodynamics
}

\author{
Shin-ichi Sasa $\S$ \\ Department of Pure and Applied Sciences, University of Tokyo, Komaba, Tokyo \\ 153-8902, Japan
}

\begin{abstract}
We study a large deviation functional of density fluctuation by analyzing stochastic non-linear diffusion equations driven by the difference between the densities fixed at the boundaries. By using a fundamental equality that yields the fluctuation theorem, we first relate the large deviation functional with a minimization problem. We then develop a perturbation method for solving the problem. In particular, by performing an expansion with respect to the average current, we derive the lowest order expression for the deviation from the local equilibrium part. This expression implies that the deviation is written as the space-time integration of the excess entropy production rate during the most probable process of generating the fluctuation that corresponds to the argument of the large deviation functional.

PACS numbers: 05.10.Gg, 05.70.Ln, 05.10.-a
\end{abstract}

\section{Introduction}

The nature of macroscopic fluctuations in equilibrium systems is determined by thermodynamics. Mathematically, this implies that the large deviation functional for the fluctuations is given by a thermodynamic function. This relation is called Einstein's formula, and it is an important consequence of equilibrium statistical mechanics.

Apart from thermodynamic systems, there are many systems in which macroscopic fluctuations are studied. In general, it appears sound to consider that properties of macroscopic fluctuations for non-equilibrium systems depend on the systems under consideration. However, from an optimistic viewpoint, it may be expected that there exists a class of non-equilibrium systems in which the large deviation functional has a physical correspondence with macroscopic deterministic laws such as those in thermodynamics and hydrodynamics.

Recently, researches speculating the existence of such a system have been presented. In one approach, an extended form of thermodynamics is explored before considering fluctuations [1]. If it is operationally and consistently constructed, this extended function might provide a large deviation functional. Although there are some successful

$\S$ To whom correspondence should be addressed (sasa@jiro.c.u-tokyo.ac.jp). 
examples [2, 3], it turned out a severe restriction is required to extend a thermodynamic framework in macroscopic systems [3].

Another approach utilized a new variation principle called the additivity principle [4, 5, 6]. Indeed, with the aid of this principle, non-trivial forms of large deviation functionals were exactly derived for some models. Although the discovery of this principle stimulates us to expect it to possess generality, there is no evidence that it is useful for a wide class of non-equilibrium systems.

Considering the above-mentioned difficulties in constructing a general framework, we have decided that concrete expressions of large deviation functionals should be investigated for several models. With this motivation, we attempt to develop a perturbation method for calculating large deviation functionals. In particular, we analyze a fluctuating hydrodynamic model that describes the density fluctuations in systems that are attached to particle reservoirs at the boundaries (see Sec. 21). Even for this simple model, a perturbation method has never been proposed, except for the approximation method by which large deviation functionals are treated as a quadratic form [7]. This approximate expression can be obtained by the analysis of a linearized equation, but it is not appropriate for our purpose because we wish to know the physical correspondence of large deviation functionals.

In this paper, we expand a large deviation functional with respect to the average current, maintaining the nature of large fluctuations. Here, it should be noted that the large deviation functional can be exactly derived for equilibrium cases. This exact derivation is due to a detailed balance property. Thus, in order to develop a perturbation method with respect to the average current, we need to find a useful relation that corresponds to an extended version of the detailed balance property. Fortunately, a fundamental relation that recovers the detailed balance condition in equilibrium cases is known to be related to the fluctuation theorem [8, 9, 10, 11, 12]. Using this relation, in Sec. 3, we formulate a minimization problem to which a perturbation method can be applied for determining the large deviation functional. In Sec. 4, we calculate the large

deviation functional of the lowest order with respect to the average current. Section 5 is devoted to concluding remarks.

\section{Preliminaries}

\subsection{Model}

We consider a one-dimensional system in contact with particle reservoirs at the boundaries $x=0$ and $x=L$. Let $\rho(x, t)$ be the coarse-grained density field. We assume that $\rho(x, t)$ obeys the stochastic partial differential equation

$$
\partial_{t} \rho=\partial_{x}\left(D(\rho) \partial_{x} \rho+\xi\right)
$$

with Gaussian white noise $\xi$ that satisfies

$$
\langle\xi(x, t) \xi(y, s)\rangle=2 \sigma(\rho(x)) T \delta(x-y) \delta(t-s)
$$


where $D$ in (11) and $\sigma$ in (3) represent the diffusion constant and the conductivity in the system under consideration, respectively. $T$ is the temperature, and the Boltzmann constant is set to unity. It is straightforward to extend our analysis for $d(\geq 2)$ dimensional systems, but for simplicity we focus on the one-dimensional case.

Since we are interested in the large deviation functional of the density field, we set $\epsilon=1 / L$ and introduce a new space-time coordinate $\left(x^{\prime}, t^{\prime}\right)=\left(\epsilon x, \epsilon^{2} t\right)$. With this coordinate, we define a new field $\rho^{\prime}\left(x^{\prime}, t^{\prime}\right)=\rho(x, t)$ and noise $\xi^{\prime}\left(x^{\prime}, t^{\prime}\right)=\epsilon^{-1} \xi(x, t)$. Then, $\rho^{\prime}\left(x^{\prime}, t^{\prime}\right)$ satisfies the same equation as (11) with prime symbols. Omitting the prime symbols, we obtain (1) with

$$
\langle\xi(x, t) \xi(y, s)\rangle=2 \epsilon \sigma(\rho(x)) T \delta(x-y) \delta(t-s) .
$$

We impose the boundary conditions $\rho(0, t)=\rho_{0}$ and $\rho(1, t)=\rho_{1}$, where we assume that $\rho_{0} \leq \rho_{1}$ without loss of generality.

\subsection{Notation}

Since a few types of functional dependence appear in this paper, we use the following notations in order to avoid confusion. Formally, $\rho$ represents the density field as a function of $(x, t)$, where $0 \leq x \leq 1$ and $0 \leq t \leq \infty$. However, as in the usual practice in physics, we write $\rho(x, t)$ as $\rho$ if the argument can be easily guessed. On the contrary, when a quantity $g$ depends on the function $\rho$, we explicitly write the functional dependence as $g(\rho())$. Thus, for example, $D(\rho)$ in (1) does not represent the functional dependence of $\rho$, but it should be read as $D(\rho(x, t))$. Furthermore, the density field as a function of $x$ is denoted by $\hat{\rho}$. In a similar manner, $h(\hat{\rho})$ represents $h(\hat{\rho}(x))$ and $g(\hat{\rho}())$ represents the functional dependence of $\hat{\rho}$. We also use the expression $\rho(, 0)=\hat{\rho}()$ as an identity for functions of $x$. That is, this means that $\rho(x, 0)=\hat{\rho}(x)$ for any value of $x$.

\subsection{Question}

We study statistical properties in the steady state of this model. The average current $\bar{J}$ and average density profile $\bar{\rho}(x)$ are obtained from the relation

$$
D(\bar{\rho}) \partial_{x} \bar{\rho}=-\bar{J}
$$

with the boundary conditions $\bar{\rho}(0)=\rho_{0}$ and $\bar{\rho}(1)=\rho_{1}$. However, it has been known that fluctuations around the average value exhibit non-trivial behaviors such as non-local correlations [7]. Here, let $P_{\mathrm{s}}(\hat{\rho}())$ be the stationary distribution of density fluctuations. In the limit $\epsilon \rightarrow 0(L \rightarrow \infty$ in the original problem), it is characterized by the leading order expression

$$
\left.P_{\mathrm{s}}(\hat{\rho}()) \simeq \exp \left[-\frac{1}{\epsilon} I(\hat{\rho}())\right)\right],
$$

where $I(\hat{\rho}())$ is called the large deviation functional. 
Despite the simplicity of the model, it is quite difficult to calculate the large deviation functional for general non-equilibrium cases. To date, only for the special case where $D=1$ and $\sigma(\rho)=\rho(1-\rho)$, it was derived as a variational form by using the Hamilton-Jacobi formulation for the model described by (11) with (3) [13]. It should be noted that this large deviation functional is identical to the exact solution for a simple exclusion process in contact with particle reservoirs at the boundaries [4]. However, we find that the special technique for solving the Hamilton-Jacobi equation cannot be used for other functional forms of $D(\rho)$ and $\sigma(\rho)$.

Here, let us consider the problem from a physical viewpoint. We first review the result for the equilibrium case $\rho_{0}=\rho_{1}$. According to Einstein's formula, the large deviation functional is written as

$$
I(\hat{\rho}())=\beta[F(\hat{\rho}())-F(\bar{\rho}())]
$$

with

$$
F(\hat{\rho}())=\int_{0}^{1} d x[f(\hat{\rho}(x))-\mu \hat{\rho}(x)]
$$

where $\beta$ is the inverse temperature; $f(\rho)$, the free energy density; $\mu$, the chemical potential defined by $\mu=f^{\prime}\left(\rho_{0}\right)$. Hereafter, the prime represents the derivative with respect to the density. Furthermore, with regard to the intensity of density fluctuations $\chi\left(=\left\langle\left[\int_{0}^{1} d x\left(\rho(x, t)-\rho_{0}\right)\right]^{2}\right\rangle / \epsilon\right)$, we know that the equality $f^{\prime \prime}=T \chi^{-1}$ and the Einstein relation $D \chi=\sigma T$ hold (see the review in Ref. [14). These lead to

$$
f^{\prime \prime}(\rho)=\frac{D(\rho)}{\sigma(\rho)} .
$$

In this manner, we have determined the large deviation functional without any calculation. On the contrary, without any physical consideration, we can derive the large deviation functional in the form of (6) using (7) and (8). (See the argument below (24) in Sec. 3.2.)

The physical argument developed above cannot be applied to non-equilibrium cases $\left(\rho_{0}<\rho_{1}\right)$ because thermodynamics for non-equilibrium steady states has not yet been established. Nevertheless, it is expected that the large deviation functional for nonequilibrium cases might have a correspondence with the local equilibrium form, where the function $F()$ given in (7) is replaced with

$$
F_{\mathrm{le}}(\hat{\rho}())=\int_{0}^{1} d x[f(\hat{\rho}(x))-\mu(x) \hat{\rho}(x)] .
$$

The chemical potential in this expression depends on $x$, and its functional form is given by the local equilibrium thermodynamics. That is,

$$
\mu(x)=f^{\prime}(\bar{\rho}(x)) .
$$

Further, considering the relation

$$
D(\rho) \partial_{x} \rho=\sigma(\rho) \partial_{x} f^{\prime}(\rho)
$$


derived from (8), we obtain

$$
\bar{J}=-\sigma(\bar{\rho}) \partial_{x} \mu \text {. }
$$

This implies that $\sigma$ is indeed the conductivity.

Here, it should be noted that the large deviation function is not equal to $\beta\left[F_{\text {le }}(\hat{\rho}())-F_{\text {le }}(\bar{\rho}())\right]$, but there is a deviation from the local equilibrium part. We then express the function $F$ in (6) as

$$
F(\hat{\rho}())=F_{\mathrm{le}}(\hat{\rho}())+N(\hat{\rho}()) .
$$

At present, the specific objective of this study is to find a physical interpretation of $N(\hat{\rho}())$ by obtaining its expression.

\section{Formal analysis}

\subsection{Fundamental equality}

We first define

$$
F_{0}(\hat{\rho}())=\int_{0}^{1} d x f(\hat{\rho}(x))
$$

where $f$ is given in (8). We then rewrite (1) as the continuity equation

$$
\partial_{t} \rho+\partial_{x} j=0
$$

with

$$
j=-\sigma(\rho) \partial_{x} \frac{\delta F_{0}}{\delta \rho(x)}+\xi
$$

where we have used (11). Since $\xi$ obeys the Gaussian process, the probability distribution of $\rho(, t), 0<t \leq \tau$, with fixed $\rho(, 0)$, is written as

$$
\mathcal{P}(\rho()) \simeq \exp \left[-\frac{\beta}{4 \epsilon} \int_{0}^{\tau} d t \int_{0}^{1} d x \frac{1}{\sigma(\rho)}\left(j+\sigma(\rho) \partial_{x} \frac{\delta F_{0}}{\delta \rho(x)}\right)^{2}\right],
$$

where the current $j$ is connected with $\rho$ by the continuity equation given in (15). Note that the so-called Jacobian term does not appear in the leading order in the limit $\epsilon \rightarrow 0$.

We next assume that the configuration $\hat{\rho}$ at $t=0$ obeys the local equilibrium distribution

$$
P_{\mathrm{le}}(\hat{\rho}()) \simeq \exp \left[-\frac{\beta}{\epsilon}\left(F_{\mathrm{le}}(\hat{\rho}())-F_{\mathrm{le}}(\bar{\rho}())\right)\right] .
$$

Let $A(\rho())$ be an arbitrary function of $\rho()$, and let $\langle A\rangle$ represent the average of $A$ with respect to the path probability distribution $P_{\mathrm{le}}(\hat{\rho}) \mathcal{P}(\rho())$.

In the equilibrium case $\left(\rho_{0}=\rho_{1}\right)$, the model satisfies the detailed balance condition. This property is also known as stochastic reversibility, and it can be formalized by introducing the time-reversed trajectory of $\rho$ that is denoted by $\rho^{\dagger}$. This can be explicitly written as $\rho^{\dagger}(x, t)=\rho(x, \tau-t)$. In terms of $\rho^{\dagger}$, the stochastic reversibility is expressed as a symmetry property $\langle A\rangle=\left\langle A^{\dagger}\right\rangle$, where $A^{\dagger}(\rho()) \equiv A\left(\rho^{\dagger}()\right)$. The equality $\langle A\rangle=\left\langle A^{\dagger}\right\rangle$ is 
not valid for non-equilibrium systems. We then attempt to derive its extended relation. The key equality for deriving this relation is as follows:

$$
\begin{aligned}
\frac{\mathcal{P}(\rho())}{\mathcal{P}\left(\rho^{\dagger}()\right)}= & \exp \left(-\frac{\beta}{\epsilon}\left[F_{0}(\rho(, \tau))-F_{0}(\rho(, 0))\right.\right. \\
& \left.\left.+\int_{0}^{\tau} d t(\mu(1) j(1, t)-\mu(0) j(0, t))\right]\right),
\end{aligned}
$$

which is obtained by direct calculation. Using this, we calculate

$$
\begin{aligned}
\langle A\rangle & =\int \mathcal{D} \rho P_{\mathrm{le}}(\rho(, 0)) \mathcal{P}(\rho()) A(\rho()) \\
& =\int \mathcal{D} \rho P_{\mathrm{le}}(\rho(, 0)) \mathcal{P}(\rho()) \frac{P_{\mathrm{le}}\left(\rho^{\dagger}(, 0)\right)}{P_{\mathrm{le}}(\rho(, 0))} \frac{\mathcal{P}\left(\rho^{\dagger}()\right)}{\mathcal{P}(\rho())} A^{\dagger}(\rho()) \\
& =\left\langle A^{\dagger} \exp \left(\frac{\beta}{\epsilon} \int_{0}^{\tau} d t \int_{0}^{1} d x\left(\partial_{x} \mu\right) j\right)\right\rangle .
\end{aligned}
$$

Since the relation $\langle A\rangle=\left\langle A^{\dagger}\right\rangle$ is derived in the equilibrium case $\left(\partial_{x} \mu=0\right)$, (20) is regarded as an extended form of the stochastic reversibility. It has been known that this equality yields many non-trivial relations, including the Green-Kubo relation, Kawasaki's non-linear response relation, and the fluctuation theorem [12].

\subsection{Stationary distribution}

Following the method presented in Ref. [12], we derive an expression for the stationary distribution $P_{\mathrm{s}}(\hat{\rho}())$ based on the relation in (20) . The probability distribution of the configuration $\hat{\rho}$ is given by

$$
P_{\mathrm{s}}(\hat{\rho}())=\lim _{\tau \rightarrow \infty}\langle\delta(\rho(, \tau)-\hat{\rho}())\rangle .
$$

Substituting $A(\rho())=\delta(\rho(, \tau)-\hat{\rho}())$ in (20) $)$, we obtain

$$
P_{\mathrm{S}}(\hat{\rho}())=P_{\mathrm{le}}(\hat{\rho}()) \int_{\rho(, 0)=\hat{\rho}()} \mathcal{D} \rho \mathrm{e}^{-\frac{\beta}{4 \epsilon} \Sigma(\rho())},
$$

with

$$
\Sigma(\rho())=\int_{0}^{\infty} d t \int_{0}^{1} d x\left[\frac{1}{\sigma(\rho)}\left(j+D(\rho) \partial_{x} \rho\right)^{2}-4 j\left(\partial_{x} \mu\right)\right] .
$$

Substituting (6), (13), and (18) into (22), and applying the limit $\epsilon \rightarrow 0$, we obtain

$$
N(\hat{\rho}())=\frac{\beta}{4} \min _{\rho() ; \rho(, 0)=\hat{\rho}()} \Sigma(\rho()) .
$$

In equilibrium cases, using the condition $\partial_{x} \mu=0$, we can trivially solve the minimization problem as $N(\hat{\rho}())=0$ because $\Sigma(\rho()) \geq 0$ for any $\rho()$ and the solution of the diffusion equation with $\rho(, 0)=\hat{\rho}()$ yields $\Sigma=0$. On the contrary, the minimization problem is not easily solved in non-equilibrium cases, as shown below. Note that the expression of the large deviation functional in terms of the minimization over trajectories was presented in Ref. [13, 15, 16]. However, to the best of our knowledge, the expression of $N(\hat{\rho}())$ given in (24) has never been reported. 
In order to determine the trajectory minimizing $\Sigma$ on the right-hand side of (24), we consider the variation $\rho(x, t) \rightarrow \rho(x, t)+\delta \rho(x, t)$. We first assume that the trajectory $\rho()$ that minimizes $\Sigma(\rho())$ satisfies

$$
\lim _{t \rightarrow \infty} \rho(x, t)=\bar{\rho}(x) .
$$

Further, defining a new variable

$$
\partial_{x} u=\left(j+D(\rho) \partial_{x} \rho\right) / \sigma(\rho),
$$

we calculate $\delta \Sigma=\Sigma(\rho()+\delta \rho())-\Sigma(\rho())$ as

$$
\delta \Sigma=\int_{0}^{\infty} d t \int_{0}^{1} d x\left\{\left[-\sigma^{\prime}(\rho)\left(\partial_{x} u\right)^{2}-2 D(\rho)\left(\partial_{x}^{2} u\right)\right](\delta \rho)+2\left(\partial_{x} u\right)(\delta j)\right\} .
$$

From (15), $\delta j$ is related to $\delta \rho$ in the form

$$
\delta j(x, t)=-\partial_{t} \partial_{x} \int d y \Delta^{-1}(x, y) \delta \rho(y, t),
$$

where $\Delta^{-1}(x, y)$ is the Green function satisfying $\partial_{x}^{2} \Delta^{-1}(x, y)=\delta(x-y)$, and $\Delta^{-1}(0, y)=$ $\Delta^{-1}(1, y)=0$. The substitution of (28) into the variational equation $\delta \Sigma=0$ yields

$$
2 \int_{0}^{1} d y\left(\partial_{y} \Delta^{-1}(y, x)\right) \partial_{t} \partial_{y} u(y, t)=\sigma^{\prime}(\rho)\left(\partial_{x} u\right)^{2}+2 D(\rho) \partial_{x}^{2} u .
$$

To this point, $u(0, t)$ and $u(1, t)$ can be any function of time. We here assume that the trajectory minimizing $\Sigma$ is obtained in a class of trajectories that satisfy

$$
u(0, t)=u_{0} \quad \text { and } \quad u(1, t)=u_{1},
$$

where $u_{0}$ and $u_{1}$ are constants in time. Then, (29) becomes

$$
\partial_{t} u=-\sigma^{\prime}(\rho)\left(\partial_{x} u\right)^{2} / 2-D(\rho) \partial_{x}^{2} u .
$$

Furthermore, (15) and (26) lead to

$$
\partial_{t} \rho=\partial_{x}\left(D(\rho) \partial_{x} \rho-\sigma(\rho) \partial_{x} u\right) .
$$

In this manner, with the two assumptions (25) and (30), we have found that the trajectory minimizing $\Sigma$ satisfies a set of equations (31) and (32). Here, it should be noted that the difference between (17) and (23) is the term $j \partial_{x} \mu$ in (23) and that the variational equation for (23) does not depend on this term. Thus, (31) and (32) also determine the most probable trajectory under given boundary conditions in the limit $\epsilon \rightarrow 0$. Indeed, (31) and (32) are identical to the Hamiltonian equation providing the most probable trajectories [13].

Using this property, we consider the minimization problem. First, let $\rho^{\mathrm{F}}$ be the solution of the deterministic equation $\partial_{t} \rho=\partial_{x}\left(D(\rho) \partial_{x} \rho\right)$ with $\rho(x, 0)=\hat{\rho}(x)$. Then, $(\rho, u)=\left(\rho^{\mathrm{F}}\right.$, const. $)$ is one solution of the variational equation given in (31) and (32). This solution describes the most probable relaxation behavior, provided that $\rho(, 0)=\hat{\rho}()$, but it yields $\Sigma \rightarrow \infty$ unless $\partial_{x} \mu=0$. (See (23).) Therefore, this most probable trajectory is not relevant in the minimization problem. 
In order to seek another solution, we further assume that the trajectory minimizing $\Sigma$ satisfies

$$
\lim _{t \rightarrow \infty} u(x, t)=u_{*}(x) .
$$

Then, $j$ approaches $j_{*}$ in this limit, where

$$
j_{*}=-D(\bar{\rho}) \partial_{x} \bar{\rho}+\sigma(\bar{\rho}) \partial_{x} u_{*}
$$

Substituting this expression into (31), we obtain $j_{*}= \pm \bar{J}$. When $j_{*}=\bar{J}$, we derive $\partial_{x} u_{*}=0$, which corresponds to the case $\rho=\rho^{\mathrm{F}}$. We thus pay attention to the solution for the case $j_{*}=-\bar{J}$. Then, (34) leads to

$$
\partial_{x} u_{*}(x)=2 \partial_{x} \mu,
$$

and we can easily confirm that $\Sigma<\infty$ for this solution.

The physical interpretation of this solution is obtained by considering the equality $j_{*}=-\bar{J}$. Let $\rho^{\mathrm{B}}(x, t)=\rho(x,-t)$ for the solution. Then, the current for $\rho^{\mathrm{B}}$ is $\bar{J}$ at $t=-\infty$. Further, defining

$$
u^{\mathrm{B}}(x, t)=-u(x,-t)+2 f^{\prime}(\rho(x,-t)),
$$

we can directly confirm that $\left(\rho^{\mathrm{B}}, u^{\mathrm{B}}\right)$ satisfies (31) and (32) with the condition $\left(\rho^{\mathrm{B}}, u^{\mathrm{B}}\right) \rightarrow(\bar{\rho}(x), 0)$ as $t \rightarrow-\infty$. Thus, $\rho^{\mathrm{B}}(x, t)$ describes the most probable process of generating the fluctuation $\hat{\rho}()$ at $t=0$ starting from $\bar{\rho}(x)$ at $t=-\infty$. Note that the boundary values $u^{\mathrm{B}}(0, t)=u^{\mathrm{B}}(1, t)=0$ are consistent with the condition imposed in Ref. [13].

\section{Perturbation theory}

\subsection{Idea}

Before developing a perturbation theory, we consider a numerical method by which we can calculate $N(\hat{\rho}())$. First of all, we must solve (31) and (32) under the conditions $(\rho, u) \rightarrow\left(\bar{\rho}, u_{*}\right)$ as $t \rightarrow \infty$ and $\rho(, 0)=\hat{\rho}()$. Note that $u(x, 0)$ should be determined such that $(\rho, u) \rightarrow\left(\bar{\rho}, u_{*}\right)$ as $t \rightarrow \infty$. However, it is quite difficult to perform a shooting method for partial differential equations.

Instead of such a direct method, we devise the following recursive procedure. Initially, we set

$$
u^{(0)}(x, t)=u_{*}(x) .
$$

Then, for $n \geq 0$, we numerically solve

$$
\partial_{t} \rho^{(n)}=\partial_{x}\left(D\left(\rho^{(n)}\right) \partial_{x} \rho^{(n)}-\sigma\left(\rho^{(n)}\right) \partial_{x} u^{(n)}\right)
$$

with $\rho^{(n)}(x, 0)=\rho(x)$, and next we consider

$$
\partial_{t} u^{(n+1)}=-\sigma^{\prime}\left(\rho^{(n)}\right)\left(\partial_{x} u^{(n+1)}\right)^{2} / 2-D\left(\rho^{(n)}\right) \partial_{x}^{2} u^{(n+1)},
$$


with $u^{(n+1)}(x, \infty)=u_{*}(x)$. Here, solving (39), we set $\tilde{u}(x,-t)=u(x, t)$. Then, $\tilde{u}$ satisfies

$$
\partial_{t} \tilde{u}^{(n+1)}=\sigma^{\prime}\left(\rho^{(n)}\right)\left(\partial_{x} \tilde{u}^{(n+1)}\right)^{2} / 2+D\left(\rho^{(n)}\right) \partial_{x}^{2} \tilde{u}^{(n+1)},
$$

for $-\infty \leq t \leq 0$. Because $\tilde{u}^{(n)}(x,-\infty)=u_{*}(x)$, we can have a standard algorithm of the forward time evolution starting from $u_{*}(x)$, and as the result $\tilde{u}^{(n)}(x, 0)=u^{(n)}(x, 0)$ is determined. If we confirm the convergence $\rho^{(n)}(x, t) \rightarrow \rho(x, t)$ and $u^{(n)}(x, t) \rightarrow u(x, t)$ within the desired numerical accuracy, we obtain an approximate solution for (31) and (32) under the conditions $(\rho, u) \rightarrow\left(\bar{\rho}, u_{*}\right)$ as $t \rightarrow \infty$ and $\rho(, 0)=\hat{\rho}()$.

\subsection{Perturbative expansion}

The method we considered above is also useful for developing a perturbation theory. As the simplest example, we derive $N(\hat{\rho}())$ up to the order of $\bar{J}^{2}$. Checking the $\bar{J}$ dependence of $N$ given in (24), we find that the $O\left(\bar{J}^{2}\right)$ contribution can be ignored in the estimation of $u$ and $j$. Considering this, we investigate the recursion equation. First, we have $u^{(0)}(x, t)=u_{*}(x)$ and

$$
\partial_{t} \rho^{(0)}=\partial_{x}\left(D\left(\rho^{(0)}\right) \partial_{x} \rho^{(0)}-\sigma\left(\rho^{(0)}\right) \partial_{x} u_{*}\right)
$$

with $\rho^{(0)}(x, 0)=\hat{\rho}(x)$. Next, we solve

$$
\partial_{t} u^{(1)}=-D\left(\rho^{(0)}\right) \partial_{x}^{2} u^{(1)}-\sigma^{\prime}\left(\rho^{(0)}\right)\left(\partial_{x} u^{(1)}\right)^{2} / 2
$$

under the condition $\lim _{t \rightarrow \infty} u^{(1)}(x, \infty)=u_{*}(x)$. Substituting $u^{(1)}(x, t)=u^{(0)}(x, t)+$ $v^{(1)}(x, t)$ into this equation and expanding in terms of $\bar{J}$, we obtain

$$
v^{(1)}(x, t)=O\left(\bar{J}^{2}\right) .
$$

Since $u^{(1)}(x, t)=u_{*}+O\left(\bar{J}^{2}\right)$, we have arrived at the fixed point solution by ignoring the $O\left(\bar{J}^{2}\right)$ contribution in $u$ and $\rho$. Substituting this solution into (23), we obtain the expression of $N(\rho())$ as

$$
N(\hat{\rho}())=\beta \int_{0}^{\infty} d t \int_{0}^{1} d x\left(\partial_{x} \mu\right)\left[D\left(\rho^{(0)}\right) \partial_{x} \rho^{(0)}-\sigma\left(\rho^{(0)}\right) \partial_{x} \mu\right]+O\left(\bar{J}^{3}\right),
$$

with (41) and $\rho^{(0)}(x, 0)=\hat{\rho}(x)$.

Now, let us recall the physical interpretation of the solution. As discussed in the last paragraph of Sec. [3, $\rho^{\mathrm{B}}(x, t)=\rho^{(0)}(x,-t)$ describes the most probable process for generating the fluctuation $\hat{\rho}()$ at $t=0$ starting from $\bar{\rho}(x)$ at $t=-\infty$. Further, from (41), it is found that $J^{B}=D\left(\rho^{B}\right) \partial_{x} \rho^{\mathrm{B}}-\sigma\left(\rho^{\mathrm{B}}\right) \partial_{x} u_{*}$ represents the particle current in this most probable process. Using this current, we rewrite $N(\hat{\rho}())$ as

$$
N(\hat{\rho}())=-\beta \int_{-\infty}^{0} d t \int_{0}^{1} d x\left(\partial_{x} \mu\right)\left[-J^{\mathrm{B}}-\sigma\left(\rho^{\mathrm{B}}\right) \partial_{x} \mu\right]+O\left(\bar{J}^{3}\right) .
$$

Here, $-\beta\left(\partial_{x} \mu\right) J^{\mathrm{B}}$ is the entropy production rate observed in the process, while $\beta \sigma\left(\rho^{\mathrm{B}}\right)\left(\partial_{x} \mu\right)^{2}$ is interpreted as the entropy production rate under the assumption that the steady state with the density $\rho^{\mathrm{B}}(x, t)$ is locally realized in a space-time point $(x, t)$. We call the latter quasi-steady entropy production. We then interpret 
$\beta\left(\partial_{x} \mu\right)\left[-J^{\mathrm{B}}-\sigma\left(\rho^{\mathrm{B}}\right) \partial_{x} \mu\right]$ as an excess entropy production ratio. With this interpretation, we can regard $N(\hat{\rho}())$ as the space-time integration of the excess entropy absorption rate during the most probable process of generating the fluctuation $\hat{\rho}()$ at $t=0$ starting from $\bar{\rho}(x)$ at $t=-\infty$. (Note the minus sign before the space-time integration.)

\subsection{Small fluctuations}

When we focus on small fluctuations, we can make another approximation by which a quantitative calculation becomes possible. Indeed, substituting $\rho(x, t)=\bar{\rho}(x)+\phi(x, t)$ into (44), we obtain

$$
N(\hat{\rho}())=-\frac{\bar{J}^{2} A\left(\rho_{0}\right)}{2 \sigma\left(\bar{\rho}_{0}\right)^{2}} \int_{0}^{\infty} d t \int_{0}^{1} d x(\phi(x, t))^{2}+O\left(\bar{J}^{3}, \phi^{3}\right),
$$

with

$$
A\left(\rho_{0}\right)=\sigma^{\prime \prime}\left(\rho_{0}\right)-\sigma^{\prime}\left(\rho_{0}\right) D^{\prime}\left(\rho_{0}\right) / D\left(\rho_{0}\right) .
$$

Furthermore, since we ignore the terms of $O\left(\bar{J}^{3}\right)$ in $N$, we can assume that $\phi$ satisfies $\partial_{t} \phi=\partial_{x}\left(D\left(\rho_{0}\right) \partial_{x} \phi\right)$ with the initial condition $\phi(x, 0)=\hat{\rho}(x, t)-\bar{\rho}$ and the boundary conditions $\phi(0, t)=\phi(1, t)=0$. Here, considering that $\rho_{1}-\rho_{0} \simeq O(\bar{J})$, we find that $\rho_{0}$ in (46) and the boundary conditions can be replaced with an arbitrary value in $\left[\rho_{0}, \rho_{1}\right]$. This approximation leads to

$$
P_{\mathrm{s}}(\hat{\rho}()) \simeq \exp \left[-\frac{\beta}{2 \epsilon} \int_{0}^{1} d y \int_{0}^{1} d y^{\prime} L\left(y, y^{\prime}\right) \phi(y) \phi\left(y^{\prime}\right)\right] .
$$

Here, we have defined

$$
L\left(y, y^{\prime}\right)=\frac{D(\bar{\rho}(y))}{\sigma(\bar{\rho}(y))} \delta\left(y-y^{\prime}\right)-\frac{\bar{J}^{2} A\left(\rho_{0}\right)}{\sigma\left(\rho_{0}\right)^{2}} \int_{0}^{\infty} d t \int_{0}^{1} d x G(x, y, t) G\left(x, y^{\prime}, t\right),
$$

where $G(x, y, t)$ is the Green function that satisfies

$$
\left[\partial_{t}-D\left(\rho_{0}\right) \partial_{x}^{2}\right] G(x, y, t)=0
$$

when $t>0, G(x, y, 0)=\delta(x-y)$, and $G(0, y, t)=G(1, y, t)=0$. An expression that is essentially the same as that in (49) was derived by the analysis of the linearized equation around the steady solution $\bar{\rho}[7$.

Furthermore, note that the space-time integration appearing in (49) is calculated as

$$
\int_{0}^{\infty} d t \int_{0}^{1} d x G(x, y, t) G\left(x, y^{\prime}, t\right)=\frac{y^{\prime}(1-y)}{2 D\left(\rho_{0}\right)} \theta\left(y-y^{\prime}\right)+\left(y \leftrightarrow y^{\prime}\right),
$$

where $\theta()$ is Heaviside's step function. Then, from the Gaussian nature of the fluctuations, we derive

$$
\begin{aligned}
\left\langle\phi(y) \phi\left(y^{\prime}\right)\right\rangle= & \epsilon T \frac{\sigma(\bar{\rho}(y))}{D(\bar{\rho}(y))} \delta\left(y-y^{\prime}\right) \\
& +\epsilon T \frac{\bar{J}^{2} A\left(\rho_{0}\right)}{D\left(\rho_{0}\right)^{3}}\left[\frac{y^{\prime}(1-y)}{2} \theta\left(y-y^{\prime}\right)+\left(y \leftrightarrow y^{\prime}\right)\right] .
\end{aligned}
$$


In order to present a simpler demonstration, we calculate the intensity of the fluctuations of the spatially averaged density

$$
\chi=\frac{1}{\epsilon}\left\langle\left(\int_{0}^{1} d x \phi(x, t)\right)^{2}\right\rangle .
$$

Using (52), we obtain

$$
\chi=T \int_{0}^{1} d x \frac{\sigma(\bar{\rho}(x))}{D(\bar{\rho}(x))}+T \frac{\bar{J}^{2} A\left(\rho_{0}\right)}{24 D\left(\rho_{0}\right)^{3}} .
$$

The first term on the right-hand side represents the contribution of local equilibrium fluctuations, and the second term originates from non-local fluctuations. For the special case $D=1, \sigma=\rho(1-\rho)$, and $T=1$, the second term becomes $-\left(\rho_{1}-\rho_{0}\right)^{2} / 12$, which is consistent with the result obtained from the exact solution in Ref. [4].

\section{Concluding remarks}

We have derived the large deviation functional for a simple model using fluctuating hydrodynamics. In particular, focusing on the deviation $N(\hat{\rho}())$ from the local equilibrium part, we obtain its lowest order expression with respect to the average current $\bar{J}$. This expression provides us with the physical interpretation that $N(\hat{\rho}())$ corresponds to the excess entropy absorption during the most probable process of generating the fluctuation $\hat{\rho}()$ at $t=0$ starting from $\bar{\rho}(x)$ at $t=-\infty$.

The evolution equation describing the most probable process generating the fluctuation $\hat{\rho}()$ at $t=0$ is called the adjoint hydrodynamic equation. Within our approximation, we have obtained the adjoint hydrodynamic equation as (41). In contrast to equilibrium cases, the generating process is not given by the time reversal of a relaxation process owing to the existence of the second term in (41). Rather, it may be noticed that (41) is similar to the equation for driven diffusion systems. It is an interesting subject to elucidate the physical picture for this asymmetry.

Our results can be generalized to those for several cases in a straightforward manner. For example, the calculation of the large deviation functionals for driven diffusive systems may be a natural problem, which will be studied in a similar manner. Furthermore, the analysis of fluctuating hydrodynamics for a simple fluid is a physically important problem [15, 17]. With regard to this problem, it has recently been found that the stationary distribution for non-equilibrium systems with multiple heat reservoirs is expressed in terms of the excess heat up to the order of the square of the average heat flux [18]. The connection between the two results will be explored.

Before concluding this paper, let us recall again that large deviation functionals in equilibrium cases are expressed by a thermodynamic function. Thus, we are naturally led to consider a thermodynamic framework consistent with the expression of the large deviation functional that we have calculated in this paper. For example, by using a method similar to that developed in Ref. [19, we can derive an identity for the operations expressed by a parameter change. Applying the identity to the case in which 
the boundary conditions are changed, one may find some insight related to an extended framework of thermodynamics.

Furthermore, we wish to highlight the fact that the additivity of the system is an important property in a thermodynamic framework [3]. However, the additivity at a thermodynamic level is not directly related to the additivity of large deviation functionals in non-equilibrium steady states because of the existence of non-local fluctuations. Therefore, one may study the additivity principle that was discovered for exactly solvable models [4, 5, 6] from the viewpoint of an extended framework of thermodynamics. The concrete expression given in (45) will help us to consider such a problem.

\section{Acknowledgments}

The author thanks T. S. Komatsu and H. Tasaki for related discussions. This work was supported by a grant (No. 19540394) from the Ministry of Education, Science, Sports and Culture of Japan.

\section{References}

[1] Oono Y and Paniconi M 1998 Prog. Theor. Phys. Suppl. 13029

[2] Hayashi K and Sasa S 2003 Phys. Rev. E 68 035104(R)

[3] Sasa S and Tasaki H 2006 J. Stat. Phys. 125128

[4] Derrida B, J. L. Lebowitz J L, and Speer E R 2001 Phys. Rev. Lett. 87 150601; 2002 J. Stat. Phys. 107599

[5] Derrida B, Lebowitz J L, and Speer E R 2002 Phys. Rev. Lett. 89 030601; 2003 J. Stat. Phys. 110 775

[6] Bodineau T and Derrida B 2004 Phys. Rev. Lett. 92180601

[7] Spohn H 1983 J. Phys. A: Math. Gen. 164275

[8] Evans D J, Cohen E G D, and Morris D P 1993 Phys. Rev. Lett. 712401

[9] Gallavotti G and Cohen E G D 1995 Phys. Rev. Lett. 742694

[10] Kurchan J 1998 J. Phys. A: Math. Gen. 313719

[11] Maes C 1999 J. Stat. Phys. 95367

[12] Crooks G E 2000 Phys. Rev. E 612361

[13] Bertini L, Sole A De, Gabrielli D, Jona-Lasinio G and Landim C 2001 Phys. Rev. Lett. 87040601 ; 2002 J. Stat. Phys. 107635

[14] Hayashi K and Sasa S 2006 Physica A 370407

[15] Eyink G 1990 J. Stat. Phys. 61533

[16] Eyink G, Lebowitz J L, and Spohn H 1996 J. Stat. Phys. 83385

[17] Schmitz R 1988 Phys. Rep. 1711

[18] Komatsu T S and Nakagawa N 2007 in preparation

[19] Hatano T and Sasa S 2001 Phys. Rev. Lett. 863463 